\section{AVALIAÇÃO DA EFETIVIDADE DO ACOMPANHAMENTO FARMACOTERAPÊUTICO NO CONTROLE DO DIABETES MELLITUS TIPO 2 EM LONGO PRAZO}

\section{EVALUATION OF THE EFFECTIVENESS OF \\ PHARMACOTHERAPY FOLLOW-UP IN LONG-TERM CONTROL OF DIABETES MELLITUS TYPE 2}

\author{
Lucas Borges Pereira' ${ }^{1}$, Camilo Molino Guidoni², \\ Anna Paula de Sá Borges ${ }^{3}$, Leonardo Régis Leira Pereira ${ }^{1}$
}

\section{RESUMO}

Introdução: O número de farmacêuticos que atuam em unidades básicas de saúde é reduzido, dificultando a execução do acompanhamento farmacoterapêutico (AFT) para um elevado número de pacientes. Portanto, deve-se estabelecer um período de execução do AFT, para que muitos pacientes sejam contemplados, contudo são escassos os estudos que avaliam a continuidade do controle da doença após a alta do serviço. Dessa forma, este estudo propôs avaliar o desfecho clínico de pacientes diagnosticados com Diabetes mellitus tipo 2 (DM2) após a alta de um serviço de AFT.

Métodos: Estudo de coorte retrospectiva após estudo de intervenção de um acompanhamento farmacoterapêutico, que avaliou 64 pacientes divididos em dois grupos: estudo (participaram de um programa de AFT) e controle (não participaram do programa de AFT). Foram coletados dados clínicos e laboratoriais destes pacientes ao final do programa de AFT (baseline - março/2006 a fevereiro/2007), e nos quatro anos após o término do programa de AFT (março/2007 a agosto/2011).

Resultados: Dentre os 64 pacientes, 56 foram incluídos. Nos quatro anos posteriores ao serviço observou-se nove óbitos, sendo seis do grupo controle e três do grupo de estudo $(p=0,151)$. O grupo de estudo manteve os valores de hemoglobina glicada após AFT ( $\mathrm{HbA1c})(8,5 \%$ vs $8,0 \%, p=0,082)$, enquanto que o grupo controle reduziu os valores de hemoglobina glicada (HbA1c) $(9,1 \%$ vs $8,1 \% ; p=0,004)$. O controle da glicemia de jejum (GJ) do grupo de estudo foi mantido após quatro anos (149,5 mg/dL vs $148,8 \mathrm{mg} / \mathrm{dL}, p=0,884)$, bem como o grupo controle $(170,7 \mathrm{mg} / \mathrm{dL}$ vs $170,6 \mathrm{mg} / \mathrm{dL}$, $p=0,993)$, no entanto ao comparar os dois grupos após AFT, o grupo de estudo apresenta valor significativamente menor que o grupo controle $(p=0,047)$.

Conclusão: Apesar das diferenças obtidas entre os grupos com o AFT não permanecerem após quatro anos, os resultados clínicos e laboratoriais não apresentaram piora significativa nesse período.

Palavras-chave: Acompanhamento farmacoterapêutico; cuidado farmacêutico; diabetes mellitus; Sistema Único de Saúde; hipoglicemiantes; hemoglobina A glicosilada; glicemia

\section{ABSTRACT}

Introduction: The number of pharmacists working at primary health care units is small, which means that pharmacotherapy follow-up (PFU) cannot be offered to a high number of patients. An established period of PFU could then lead to more patients being treated. However, studies assessing management of chronic diseases after discharge from this service are scarce. Thus, this study evaluated clinical outcomes of patients diagnosed with diabetes mellitus type 2 (DM2) after PFU discharge.
Clin Biomed Res. 2018;38(3):237-244

1 Faculdade de Ciências Farmacêuticas de Ribeirão Preto, Universidade de São Paulo (USP). Ribeirão Preto, SP, Brasil.

2 Departamento de Ciências Farmacêuticas, Universidade Estadual de Londrina (UEL). Londrina, PR, Brasil.

3 Faculdade de Farmácia, Universidade Estadual de Goiás (UEG). Itumbiara, $\mathrm{GO}$, Brasil.

Autor correspondente: Lucas Borges Pereira lucas.borges.pereira@usp.br Faculdade de Ciências Farmacêuticas de Ribeirão Preto, Universidade de São Paulo (USP)

Avenida do Café, s/n. 14040-903, Ribeirão Preto, SP, Brasil. 
Methods: This retrospective cohort study was conducted after a PFU intervention study, which evaluated 64 patients divided into two groups: study (who participated in a PFU program) and control (who did not participate in a PFU program). Laboratory and clinical data were collected from these patients at the end of the program (baseline March 2006 to February 2007) and for four years after the end of the program (March 2007 to August 2011).

Results: Of 64 patients, 56 were enrolled. In four years after discharge, nine patients died, six of them were from the control group and three from the study group $(p=0.151)$. The study group maintained glycated hemoglobin A $(\mathrm{HbA} 1 \mathrm{c})$ levels after PFU $(8.5 \%$ vs. $8.0 \%, p=0.082)$, while the control group showed reduced levels $(9.1 \%$ vs. $8.1 \%$; $p=0.004)$. Fasting glucose remained under control in the study group $(149.5 \mathrm{mg} / \mathrm{dL}$ vs. $148.8 \mathrm{mg} / \mathrm{dL}, p=0.884)$ as well as in the control group $(170.7 \mathrm{mg} / \mathrm{dL}$ vs. $170.6 \mathrm{mg} / \mathrm{dL}$, $p=0.993$ ) after four years. However, when the groups were compared after PFU, the study group showed a significantly lower value than the control group $(p=0.047)$.

Conclusion: Although the differences observed between the groups during PFU did not remain after four years, clinical and laboratory results did not show significant worsening in the period.

Keywords: Pharmacotherapy follow-up; pharmaceutical care; diabetes mellitus; Brazilian Unified Health System; hypoglycemic agents; glycated hemoglobin A; blood glucose

A Cuidado Farmacêutico (CF) tem como objetivo promover o uso racional dos medicamentos, ao identificar, resolver e prevenir problemas relacionados a medicamentos, melhorando a qualidade de vida dos pacientes e o controle adequado das doenças, em especial aquelas de caráter crônico, por meio de atividades clínicas como o acompanhamento farmacoterapêutico (AFT) $)^{1,2}$.

Apesar de vários estudos publicados na literatura comprovarem a efetividade do AFT na redução dos parâmetros clínicos e laboratoriais, tais como glicemia de jejum (GJ), hemoglobina glicada (HbA1c), pressão arterial (PA) e perfil lipídico ${ }^{3-6}$, observa-se que essa atividade, sobretudo no Brasil, ainda continua sendo ofertada para um número reduzido de pacientes; existindo a necessidade do desenvolvimento de estratégias que possibilitem aumentar o número de pacientes atendidos em serviços de AFT.

Diante dessa realidade, observa-se que a razão usuários das farmácias no Sistema Único de Saúde (SUS) e profissionais farmacêuticos é desfavorável para a oferta de serviços de AFT a um número maior de indivíduos, além da dificuldade gerada pela necessidade de os farmacêuticos dedicarem-se às atividades gerenciais e administrativas da Assistência Farmacêutica, o que limita seu tempo para as atividades voltadas à tecnologia do uso dos medicamentos ${ }^{7,8}$.

Sendo assim, para atender um maior número de usuários num serviço de AFT, torna-se fundamental equilibrar a seleção dos pacientes e a alta farmacêutica. Diante disso, cabe ao profissional buscar mecanismos precisos na inclusão e na liberação dos usuários do acompanhamento, julgando que o usuário adquiriu o conhecimento necessário quanto ao uso correto dos medicamentos, cuidados à saúde em geral e controle da doença. Na literatura, observa-se que o período de AFT situa-se entre 12 e 36 meses $^{9-11}$. Os estudos que analisam o período de AFT são escassos, portanto não há definições sobre o tempo ideal de acompanhamento. Portanto, estudos que avaliem se o período de AFT realizado foi suficiente para que os pacientes mantenham o controle adequado do Diabetes mellitus tipo 2 (DM2) após a alta farmacêutica são necessários.

À vista disso, o objetivo deste estudo foi avaliar os parâmetros clínicos, laboratoriais e farmacoterapêuticos de pacientes diagnosticados com DM2, que foram acompanhados por um serviço de AFT por um período de 12 meses.

\section{MÉTODOS}

\section{Desenho e Local de Estudo}

Trata-se de um estudo de coorte retrospectiva, cuja exposição foi uma intervenção farmacoterapêutica realizada por Borges et al. ${ }^{9}$ entre 2006 e 2007 . Sendo assim, foi avaliado os mesmos 64 pacientes que participaram do estudo de Borges et al.

Esta amostra foi obtida de uma população de 994 pacientes diagnosticados com DM2 atendidos no Centro de Saúde Escola da Faculdade de Medicina de Ribeirão Preto da Universidade de São Paulo (CSE-FMRP-USP). Considerando o 
nível de significância de $5 \%$, a população alvo de 994 pacientes, a média $(1,21 \%)$ e o desvio padrão $(1,64)$ da redução de $\mathrm{HbA} 1 \mathrm{c}$, por causa do CF, encontrado em estudos que avaliam a efetividade do CF, realizou-se o cálculo amostral de acordo com o descrito por Medronho et al. ${ }^{12}$ para estudos de intervenção e encontrou o valor de 29 pessoas para cada grupo.

Deste modo, para a avaliação da situação atual desses pacientes, optou-se por manter a divisão original dos pacientes em dois grupos, controle $(n=31)$ que recebeu entre os anos de 2006 e 2007 o cuidado padrão oferecido pelo SUS (consulta médica e retirada dos medicamentos na farmácia), e estudo ( $n=33$ ), que além do cuidado padrão, foi acompanhado pelo serviço de AFT relatado no estudo de Borges et al. ${ }^{9}$ (Figura 1).

\section{Delineamento}

O presente estudo realizou o levantamento de dados de março de 2007 a agosto de 2011, referente ao período após AFT. Esses resultados foram comparados com o final do período que os pacientes receberam o AFT, entre março de 2006 e fevereiro de 2007 (baseline).

A coleta das informações ocorreu junto aos prontuários médicos dos pacientes, e no banco de dados eletrônico conhecido como sistema Hygia, no qual são registradas todas as informações referentes ao atendimento dos pacientes do município de Ribeirão

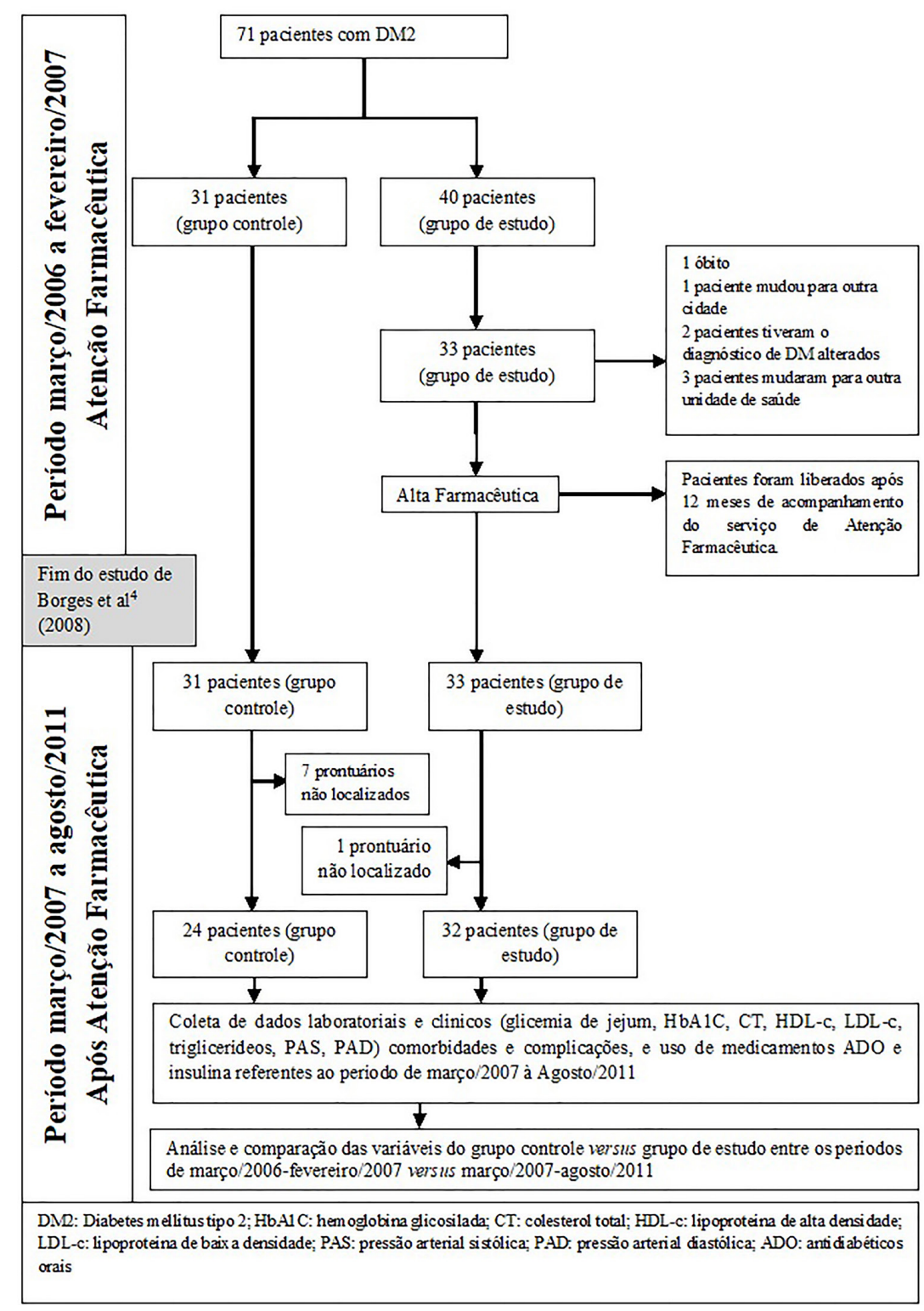

Figura 1: Fluxograma esquemático do delineamento do estudo. 
Preto (SP) no SUS. Nos prontuários médicos dos pacientes foram coletados dados como: gênero, idade, escolaridade, tempo de diagnóstico, GJ, HbA1c, pressão arterial sistólica (PAS), pressão arterial diastólica (PAD), colesterol total, lipoproteína de alta densidade (HDL-c), lipoproteína de baixa densidade (LDL-c), triglicerídeos e a presença de comorbidades (dislipidemia e hipertensão arterial) e/ou complicações [nefropatia, retinopatia, neuropatia, acidente vascular cerebral (AVC), doenças cardiovasculares (DCV), amputação, insuficiência renal]. As informações sobre os medicamentos (antidiabéticos orais e insulina) utilizados por esses pacientes no período do estudo, inclusive a retirada mensal dos medicamentos foram coletadas no sistema Hygia.

Foram excluídos do estudo os pacientes cujos prontuários não foram localizados na unidade de saúde. Além disso, os dados de pacientes que morreram durante o período de estudo foram considerados na coleta e análise dos resultados até a data do óbito.

\section{Análise de Dados}

Para a análise estatística, utilizou-se o programa Statistical Package for Social Sciences (SPSS, version 11.5, 2002). Foi realizada a comparação das variáveis dentro de cada grupo (baseline versus após AFT) e também a comparação das variáveis entre grupo controle versus grupo de estudo após a alta farmacêutica. O teste $t$ Student foi utilizado para a comparação da média das variáveis contínuas com distribuição normal, sendo que a significância estatística considerada foi $p<0,05$.

\section{Aspectos Éticos}

Este estudo foi aprovado pelo Comitê de Ética em Pesquisa da Faculdade de Ciências Farmacêuticas de Ribeirão Preto, Universidade de São Paulo, promulgado pelo protocolo $n^{\circ} 216 /$ CEP/FCFRP, conforme regem as Diretrizes e Normas Regulamentadoras de Pesquisa Envolvendo Seres Humanos do Conselho Nacional de Saúde.

\section{RESULTADOS}

Dos 64 pacientes que finalizaram o estudo realizado por Borges et al. (9), oito foram excluídos devido a indisponibilidade dos prontuários no CSE-FMRP-USP. Dessa forma foram incluídos 56 pacientes (32 do grupo de estudo e 24 do grupo controle) (Figura 1). No período após AFT foram registrados nove óbitos, sendo três do grupo de estudo e seis do grupo controle $(p=0,151)$.

Com relação à média de idade, não houve diferença estatística $(p=0,091)$ quando comparado o grupo de estudo (72,97 $\pm 8,24$ anos) versus o grupo controle (68,32 $\pm 11,30$ anos). Apesar de existir predomínio do sexo feminino em ambos os grupos ( $72 \%$ no controle e $56 \%$ no grupo de estudo), não houve diferença estatística entre eles $(p=0,403)$. O tempo de diagnóstico do DM2 nos grupos de estudo e controle foram de 17,31 $( \pm 6,30)$ e 17,64 $( \pm 7,79)$ anos, respectivamente, não observando diferença estatística $(p=0,861)$.

Durante a coleta de dados observou-se a ausência de alguns resultados de exames clínicos e laboratoriais para alguns pacientes. No grupo controle, 22 pacientes apresentaram resultados de GJ, HbA1c; 21 apresentaram resultados de colesterol total, PAS e PAD; 20 apresentaram resultados de triglicerídeos, 19 de HDL-c, e 18 de LDL-c. Enquanto que no grupo de estudo, 32 pacientes apresentaram resultados de GJ, PAS e PAD; 30 apresentaram resultados de colesterol total e triglicerídeos; e 29 apresentaram resultados de $\mathrm{HbA1c}$, HDL-c e LDL-c.

Ao avaliar se houve diferença estatística nos parâmetros clínicos e laboratoriais entre os períodos baseline e após AFT, e entre os grupos controle e estudo (Tabela 1), encontrou-se redução significativa na $\mathrm{HbA} 1 \mathrm{c}$ e no HDL-c do grupo controle, e no grupo de estudo ocorreu redução significativa no HDL-c e na PAD quando comparado o baseline ao período após AFT. Somente a GJ apresentou diferença significativa entre os grupos de estudo e controle no período após AFT.

Em relação aos medicamentos foi observado aumento significativo na dose diária de metformina em ambos os grupos, e na dose diária de glibenclamida apenas no grupo de estudo (Tabela 2), quando comparado o baseline versus após AFT. Entretanto, não houve diferença estatisticamente significativa quando se compara o grupo de estudo versus controle no período após AFT.

Observou-se que os grupos de estudo e controle não apresentaram diferença estatística quando se comparou a frequência de complicações relacionados ao DM2 no período baseline com o período após AFT. No entanto, a diferença que havia no perído baseline entre os grupos foi perdida após AFT (Tabela 3). Em relação às comorbidades, também não foi encontrada diferença estatística nos grupos de estudo e controle quando se comparou o período baseline com o período após AFT. Ao se comparar os dois grupos no período após AFT, observa-se diferença estatística entre eles, o grupo de estudo apresenta 22 pacientes com duas comorbidades, enquanto nove pacientes no grupo controle apresenta duas comorbidades. 
Tabela 1: Média ( \pm desvio padrão) dos parâmetros clínicos e laboratoriais referentes aos grupos de estudo e controle (baseline versus após AFT).

\begin{tabular}{|c|c|c|c|c|c|c|}
\hline \multirow{2}{*}{$\begin{array}{l}\text { Parâmetros clínicos e } \\
\text { laboratoriais }\end{array}$} & \multicolumn{3}{|c|}{ Grupo controle $(n=24)$} & \multicolumn{3}{|c|}{ Grupo de estudo $(n=32)$} \\
\hline & Baseline & Após AFT & $p$ valor & Baseline & Após AFT & p valor \\
\hline Glicemia de jejum (mg/dL) & $170,7( \pm 56,6)$ & $170,6( \pm 41,5)^{*}$ & 0,993 & $149,5( \pm 37,2)$ & $148,8( \pm 36,5)^{*}$ & 0,884 \\
\hline HbA1c (\%) & $9,1( \pm 1,5)$ & $8,1( \pm 1,5)$ & 0,004 & $8,5( \pm 0,8)$ & $8,0( \pm 1,8)$ & 0,082 \\
\hline Colesterol total (mg/dL) & $182,0( \pm 30,9)$ & $184,1( \pm 37,5)$ & 0,718 & $176,8( \pm 25,8)$ & $169,3( \pm 29,0)$ & 0,068 \\
\hline HDL-c (mg/dL) & $52,6( \pm 9,3)$ & $45,7( \pm 9,5)$ & 0,004 & $50,2( \pm 10,2)$ & $44,2( \pm 9,2)$ & 0,000 \\
\hline LDL-c (mg/dL) & $103,0( \pm 22,0)$ & $107,3( \pm 31,8)$ & 0,516 & $95,3( \pm 22,4)$ & $96,6( \pm 23,0)$ & 0,771 \\
\hline Triglicerídeos (mg/dL) & $150,5( \pm 68,1)$ & $156,6( \pm 79,6)$ & 0,61 & $131,9( \pm 69,2)$ & $135,3( \pm 69,2)$ & 0,65 \\
\hline PAS (mm Hg) & $139,2( \pm 20,7)$ & $136,8( \pm 17,1)$ & 0,635 & $136,4( \pm 12,8)$ & $134,1( \pm 11,6)$ & 0,154 \\
\hline PAD (mm Hg) & $82,7( \pm 9,3)$ & $80,9( \pm 7,9)$ & 0,433 & $80,1( \pm 8,0)$ & $77,2( \pm 6,9)$ & 0,004 \\
\hline
\end{tabular}

HbA1c: Hemoglobina glicosilada; HDL-c: colesterol de alta densidade; LDL-c: colesterol de baixa densidade; Após AFT: período referente após a alta da AFT; PAS: Pressão Arterial Sistólica; PAD: Pressão Arterial Diastólica. * $p=0,047$ quando comparado o grupo de estudo e o controle, no período após-AFT.

Tabela 2: Dose diária média ( \pm desvio padrão) dos antidiabéticos orais e de insulina dos grupos de estudo e controle (baseline versus após AFT).

\begin{tabular}{|c|c|c|c|c|c|c|}
\hline \multirow{2}{*}{ Dose média } & \multicolumn{3}{|c|}{ Grupo controle $(n=24)$} & \multicolumn{3}{|c|}{ Grupo de estudo ( $n=32$ ) } \\
\hline & Baseline & Após AFT & p valor & Baseline & Após AFT & p valor \\
\hline Metformina (mg/dia) & $\begin{array}{c}1479,00 \\
( \pm 1158,21)\end{array}$ & $\begin{array}{c}1758,96 \\
( \pm 1027,49)\end{array}$ & 0,021 & $\begin{array}{c}1421,09 \\
( \pm 1109,36)\end{array}$ & $\begin{array}{c}1870,36 \\
( \pm 794,35)\end{array}$ & 0,018 \\
\hline Glibenclamida (mg/dia) & $\begin{array}{c}5,40 \\
( \pm 6,60)\end{array}$ & $\begin{array}{c}5,90 \\
( \pm 5,74)\end{array}$ & 0,607 & $\begin{array}{c}5,55 \\
( \pm 6,77)\end{array}$ & $\begin{array}{c}7,08 \\
( \pm 6,97)\end{array}$ & 0,036 \\
\hline Insulina (UI/dia) & $\begin{array}{c}11,52 \\
( \pm 16,66)\end{array}$ & $\begin{array}{c}15,70 \\
( \pm 22,00)\end{array}$ & 0,094 & $\begin{array}{c}17,59 \\
( \pm 26,95)\end{array}$ & $\begin{array}{c}25,84 \\
( \pm 30,43)\end{array}$ & 0,096 \\
\hline
\end{tabular}

Tabela 3: Frequência de pacientes diagnosticados com complicações e/ou comorbidades associadas ao DM2.

\begin{tabular}{|c|c|c|c|c|c|c|}
\hline \multirow[b]{2}{*}{ Frequência de comorbidades } & \multicolumn{2}{|c|}{ Grupo controle } & \multirow{2}{*}{$\begin{array}{c}p \\
0,415\end{array}$} & \multicolumn{2}{|c|}{ Grupo de estudo } & \multirow{2}{*}{$\begin{array}{c}\boldsymbol{p} \\
0,189\end{array}$} \\
\hline & Baseline & Após AFT* & & Baseline & Após AFT* & \\
\hline Nenhuma & 2 & 4 & & 4 & 0 & \\
\hline Uma & 13 & 8 & & 8 & 9 & \\
\hline Duas & 16 & 9 & & 21 & 22 & \\
\hline Frequência de complicações & Baseline ${ }^{* *}$ & Após AFT & 0,622 & Baseline** & Após AFT & 0,066 \\
\hline Nenhuma & 10 & 7 & & 19 & 9 & \\
\hline uma ou duas & 21 & 13 & & 10 & 17 & \\
\hline três ou mais & 0 & 1 & & 4 & 5 & \\
\hline
\end{tabular}

${ }^{*} p=0,012 ;{ }^{* *} p=0,003$.

\section{DISCUSSÃO}

A HbA1c é o principal parâmetro para avaliar o controle do DM, uma vez que considera o metabolismo da glicose tanto em jejum como no período pós-prandial. Além disso, é uma medida que estima os valores glicêmicos durante o período de 120 dias anteriores a realização do exame ${ }^{10}$. De acordo com a American Diabetes Association $(A D A)^{13}$ o valor ideal de $\mathrm{HbA} 1 \mathrm{c}$ para reduzir as chances de complicações micro e macrovasculares é menor que $7,0 \%$, porém para pacientes que já possuem complicações micro e macrovasculares ou um longo tempo de DM este valor pode alcançar no máximo $8,0 \%{ }^{13}$. O grupo controle apresentou redução significativa da $\mathrm{HbA} 1 \mathrm{c}$, atingindo valores próximos ao grupo de estudo, porém permanece acima do limite permitido pela ADA. Apesar de não alcançar a significância estatística, a média dos valores do grupo de estudo também reduziu.

Uma possível justificativa para a manutenção (observado no grupo de estudo) e até redução do valor de HbA1c (observado no grupo controle) no período após AFT pode estar relacionada ao aumento significativo da dose de metformina, e acréscimo na dose de glibenclamida, embora este seja significativo apenas no grupo de estudo. Além disso, houve incremento na dose de insulina em $47 \%$ e $36 \%$, nos grupos de estudo e controle, respectivamente. 
Sabe-se que esse hormônio é indicado quando o nível de HbA1c permanece acima do valor desejado, mesmo após o ajuste das doses de antidiabéticos orais $^{14}$.

Esses resultados indicam que o grupo de estudo utiliza maior quantidade de medicamentos (não estatisticamente significativa), quando comparado ao grupo controle, para manter o controle glicêmico adequado. Porém, observa-se no trabalho de Borges et al. ${ }^{9}$ que o grupo de estudo utilizava doses maiores de glibenclamida e insulina ${ }^{9}$.

De acordo com a ADA (2013) a GJ dos pacientes com DM deve manter-se entre 70 e $130 \mathrm{mg} / \mathrm{dL}$ para reduzir os riscos de agravamentos como nefropatia, neuropatia e retinopatia ${ }^{13}$. Apesar de ambos os grupos apresentarem valores acima do recomendado pela ADA, a GJ permaneceu praticamente inalterada no período após AFT quando comparada ao baseline. Entretanto os valores são significativamente menores no grupo de estudo, quando comparado ao controle.

Pinheiro et al. ${ }^{15}$ observaram valores de GJ igual a $186,2 \mathrm{mg} / \mathrm{dL}( \pm 78,08)$ em 120 pacientes atendidos por um Serviço de Endocrinologia Hospitalar. Este resultado demonstra que além do acompanhamento médico, torna-se indispensável implementar outras ações para melhorar a adesão do paciente ao tratamento e favorecer o controle da doença ${ }^{15}$. Sriram et al. ${ }^{16}$ ressaltam a importância da AFT como uma ação complementar ao acompanhamento médico no controle do DM2, sendo que os pacientes que receberam o serviço de AFT reduziram significativamente seus valores de GJ, o que não ocorreu com o grupo controle ${ }^{16}$.

Pacientes diagnosticados com DM2 têm uma predisposição de duas a quatro vezes maior de óbito por doença cardíaca em relação a não diagnosticados com DM, e quatro vezes mais chance de ter doença vascular periférica (DVP) e AVC ${ }^{17}$. Este risco eleva-se quando o paciente apresenta perfil lipídico não controlado ${ }^{18}$. As metas para o controle do perfil lipídico para pacientes diagnosticados com DM2 encontram-se na IV Diretriz Brasileira sobre Dislipidemias e Prevenção da Aterosclerose (Sociedade Brasileira de Cardiologia) ${ }^{19}$. Os dois grupos obtiveram reduções estatisticamente significativas nos valores de HDL-C, quando comparados ao baseline, o que demonstra piora no manejo do perfil lipídico de ambos os grupos, aumentando o risco de apresentarem DVP e/ou AVC. Vale ressaltar que o valor de triglicerídeos do grupo controle está acima do recomendado, o que não se observa no grupo de estudo, porém não há diferença estatisticamente significativa quando se compara o perfil lipídico de ambos os grupos no período após AFT.
O controle da PA é importante para reduzir o risco de complicações relacionadas ao DM. No estudo da United Kingdon Prospective Diabetes Study, os pacientes diagnosticados com DM2 que mantiveram a PA próximo da normalidade reduziram em $24 \%$ o risco de desenvolverem complicações relacionadas à doença, $32 \%$ o risco de morte e $37 \%$ para doenças microvasculares ${ }^{20}$. No DM2, de acordo com a VI Diretriz Brasileira de Hipertensão Arterial (2010), os valores de PA devem ser inferiores que $130 \mathrm{mmHg}$ para a PAS e $80 \mathrm{mmHg}$ para a PAD ${ }^{21}$. Observa-se que somente o grupo de estudo reduziu significativamente a PAD, quando comparado ao baseline, mantendo-se dentro dos valores recomendados. Os demais resultados referentes ao controle da PA encontram-se acima do recomendado. Mourão et al.$^{22}$ observaram que a AFT contribuiu de forma importante para a redução significativa da PAS, comparando o grupo intervenção ao controle ${ }^{22}$

O controle adequado, principalmente do perfil glicêmico em pacientes com DM2, previne ou retarda o aparecimento de complicações ou comorbidades, como neuropatia, nefropatia, retinopatia, hipertensão arterial, dislipidemia e obesidade. Scheffel et al. ${ }^{17}$ acompanharam 927 pacientes com DM2, encontrando a presença de comorbidades em $26 \%$ dos pacientes, sendo que desses, $73 \%$ apresentavam hipertensão arterial e $64 \%$ hipercolesterolemia ${ }^{17}$. Observa-se em nosso estudo aumento das complicações e das comorbidades em ambos os grupos, comparandose o período baseline versus período após AFT. Entretanto, somente o grupo controle apresentou diferença estatisticamente significativa na presença de comorbidades entre os períodos de estudo comparados.

O DM2 é uma enfermidade crônica progressiva e degenerativa, com uma evolução negativa com aumento dos anos de diagnóstico. Além do tempo de diagnóstico, a idade do paciente é um dos fatores que interferem no controle da enfermidade ${ }^{17,23,24}$. No presente estudo, observou-se que o grupo de estudo, mesmo com idade superior ao grupo controle (diferença não significativa), apresenta melhores resultados no perfil glicêmico, lipídico e na PA, apesar de alguns parâmetros não apresentarem diferenças estatisticamente significativas, entretanto sendo importante clinicamente. Apesar de não apresentar diferença estatística $(p=0,151)$, é importante ressaltar que entre 2007 e 2011 houve menor número de óbitos no grupo de estudo. Sendo assim, o melhor resultado apresentado pelo grupo de estudo pode estar relacionado à maior utilização de antidiabéticos orais e insulina.

As limitações apresentadas por este estudo estão relacionadas ao tamanho da população se comparado 
com outros trabalhos publicados na literatura que apresentam maior quantidade de pacientes ${ }^{15,22}$. Apesar disso, a amostra apresenta tamanho adequado para a realização de inferências para a população de estudo, como demonstrado por meio do cálculo amostral. Outra limitação está baseada na impossibilidade de ter acesso ao atestado de óbito dos pacientes que faleceram neste estudo, o que nos ajudaria a concluir se o maior número de óbitos no grupo controle foi devido a um pior manejo do DM2. Além disso, como a coleta de dados foi realizada toda no prontuário dos pacientes, há o viés de informação devido ao possível erro de preenchimento do prontuário. A falta de acesso aos dados dos pacientes que não possuíam os prontuários na unidade de saúde, bem como a ausência de informações confiáveis referentes à adesão desses pacientes no período após AFT, também podem ser consideradas como limitações deste estudo.

Salvo melhor juízo, não há estudos publicados por pesquisadores brasileiros que realizaram acompanhamento de pacientes por um período prolongado, característica que fortalece este trabalho. Dessa forma, considerando o elevado número de pacientes que necessitam deste serviço, o número limitado de profissionais farmacêuticos para realizar o AFT, este estudo torna-se fundamental para entender se os pacientes que receberam alta do AFT mantêm o controle atingido. Assim, podemos entender qual o período ideal deste serviço para que consigam manter o controle da doença, além de beneficiar um número maior de pacientes por meio de uma alta adequada daqueles que receberam este serviço.
Entretanto outros estudos com objetivo de avaliar a eficiência do AFT em longo prazo são necessários para corroborar o que foi encontrado nesta pesquisa, principalmente para avaliar as condições clínicas dos pacientes após a alta do serviço de acompanhamento farmacoterapêutico.

O manejo do DM2 e a melhoria da qualidade de vida dos pacientes por meio da educação em saúde e do uso racional de medicamentos, proporcionados pelo AFT, podem ser os responsáveis pelos pacientes do grupo de estudo conseguirem manter o controle adequado da doença durante os quatro anos após a alta do AFT, pois os resultados clínicos e laboratoriais não apresentaram piora significativa nesse período. Além disso, mantiveram-se em níveis melhores quando comparado ao grupo controle desde o baseline, provavelmente devido à maior dose dos antidiabéticos orais e insulina, aliados ao maior acesso ao SUS e à melhor adesão ao tratamento farmacológico, conseguido pela participação no programa de AFT durante os anos de 2006 e 2007.

\section{Agradecimentos}

Ao financiamento do Conselho Nacional de Desenvolvimento Científico e Tecnológico (CNPq) fornecido a este estudo.

Trabalho realizado na Faculdade de Ciências Farmacêuticas de Ribeirão Preto - Universidade de São Paulo.

\section{Conflitos de Interesse}

Os autores declaram não haver conflito de interesse.

\section{REFERÊNCIAS}

1. Hepler CD, Strand LM. Opportunities and responsibilities in pharmaceutical care. Am J Hosp Pharm. 1990;47(3):533-43. PMid:2316538.

2. Obreli Neto PR, Vieira JC, Cuman RKN. Impacto da atenção farmacêutica no uso racional de antimicrobianos em uma unidade básica de saúde no interior do Estado de São Paulo. Acta Scientiarum Health Science. 2011;33(2):159-64.

3. Lyra DP, Kheir N, Abriata JP, Rocha CE, Santos CB, Pelá IR. Impact of Pharmaceutical Care interventions in the identification and resolution of drug-related problems and on quality of life in a group of elderly outpatients in Ribeirao Preto (SP), Brazil. Ther Clin Risk Manag. 2007;3(6):989-98. PMid:18516258.

4. Castro M, Fuchs F, Costa Santos M, Maximiliano P, Gus M, Beltrami Moreira L, et al. Pharmaceutical care program for patients with uncontrolled hypertension. Report of a double-blind clinical trial with ambulatory blood pressure monitoring. Am J Hypertens. 2006;19(5):528-33. http://dx.doi. org/10.1016/j.amjhyper.2005.11.009. PMid:16647628.

5. Lyra DP JR, Marcellini PS, Pelá IR. Effect of pharmaceutical care intervention on blood pressure of elderly outpatients with hypertension. Rev Bras Ciênc
Farm. 2008;44(3):451-7. http:// dx.doi.org/10.1590/S151693322008000300015 .

6. Clifford RM, Davis WA, Batty KT, Davis TM. Effect of a pharmaceutical care program on vascular risk factors in type 2 diabetes. Diabetes Care. 2005;28(4):771-6. http://dx.doi. org/10.2337/diacare.28.4.771. PMid:15793171.

7. Obreli-Neto PR, Guidoni CM, Oliveira Baldoni A, Pilger D, Cruciol-Souza JM, Gaeti-Franco WP, et al. Effect of a 36-month pharmaceutical care program on pharmacotherapy adherence in elderly diabetic and hypertensive patients. Int J Clin 
Pharm. 2011;33(4):642-9. http://dx.doi. org/10.1007/s11096-011-9518-x. PMid:21544559.

8. Romano-Lieber NS, Teixeira JJV, Farhat FCLG, Ribeiro E, Crozatti MTL, Oliveira GSAA. Revisão dos estudos de intervenção do farmacêutico no uso de medicamentos por pacientes idosos. Cad Saude Publica. 2002;18(6):1499507. http://dx.doi.org/10.1590/ S0102-311X2002000600002. PMid:12488875.

9. Borges APS, Guidoni CM, Ferreira LD, Freitas O, Pereira LRL. The Pharmaceutical care of patients with type 2 diabetes mellitus. Pharm World Sci. 2010;32(6):730-6. http://dx.doi. org/10.1007/s11096-010-9428-3. PMid:20734138.

10. Borch-Johnsen K, Colagiuri S, Balkau B, Glümer C, Carstensen B, Ramachandran $A$, et al. Creating a pandemic of prediabetes: the proposed new diagnostic criteria for impaired fasting glycaemia. Diabetologia. 2004;47(8):1396-402. http://dx.doi.org/10.1007/s00125-0041468-6. PMid:15278279.

11. Correr CJ, Pontarolo R, Wiens A, Rossignoli P, Melchiors AC, Radominski R, et al. Avaliação econômica do seguimento farmacoterapêutico em pacientes com diabetes melito tipo 2 em farmácias comunitárias. Arq Bras Endocrinol Metabol. 2009;53(7):82533. http://dx.doi.org/10.1590/ s0004-27302009000700006. PMid:19942984.

12. Medronho RA. Epidemiologia. São Paulo: Editora Atheneu, 2006. p. 298.
13. American Diabetes Association. Standards of Medical Care in Diabetes. Diabetes Care. 2013;36(Suppl 1):S11-66. http:// dx.doi.org/10.2337/dc13-S011. PMid:23264422.

14. Riddle MC. Timely initiation of basal insulin. Am J Med. 2004;116(Suppl 3A):3S-9S. http://dx.doi. org/10.1016/j.amjmed.2003.12.003. PMid:15013454.

15. Pinheiro DS, Costa CD, Rocha CR FO, Mudim CA, Reis AAS, Ghedini PC. Avaliação do nível de controle glicêmico dos pacientes diabéticos tipo 2 atendidos em um Hospital Universitário. Rev Univ Vale do Rio Verde. 2013;10(2):03-11.

16. Sriram S, Chack LE, Ramasamy R, Ghasemi A, Ravi TK, Sabzghabaee AM. Impact of pharmaceutical care on quality of life in patients with type 2 diabetes mellitus. J Res Med Sci. 2011;16(Suppl 1):S412-8. PMid:22247727.

17. Scheffel RS, Bortolanza D, Weber CS, Costa LA, Canani LH, Santos KG, et al. Prevalência de complicações micro e macrovasculares e de seus fatores de risco em pacientes com diabetes melito do tipo 2 em atendimento ambulatorial. $\mathrm{Rev}$ Assoc Med Bras. 2004;50(3):263 7. http://dx.doi.org/10.1590/ S0104-42302004000300031. PMid:15499476.

18. Goldenberg P, Schenkman S, Franco LJ. Prevalênciade diabetes mellitus: diferença de gênero e igualdade entre os sexos. Rev Bras Epidemiol. 2003;6(1):18-28. http://dx.doi.org/10.1590/S1415790X2003000100004.
19. Sociedade Brasileira de Cardiologia (SBC). IV Diretriz brasileira sobre dislipidemias e prevenção da aterosclerose: Departamento de Aterosclerose da Sociedade Brasileira de Cardiologia. Arq Bras Cardiol. 2007;88(Supl. 1):1-19.

20. United Kingdom Prospective Diabetes Study Group. Tight blood pressure control and risk of macrovascular and microvascular complications in type 2 diabetes: UKPDS 38. BMJ. 1998;317(7160):703-13. http://dx.doi. org/10.1136/bmj.317.7160.703. PMid:9732337.

21. Brasileira de Cardiologia S. (SBC), Sociedade Brasileira de Hipertensão (SBH), Sociedade Brasileira de Nefrologia (SBN). VI Diretrizes Brasileiras de Hipertensão Arterial. Arq Bras Cardiol. 2010;95(Supl. 1):151.

22. Mourão $A O$, Ferreira WR, Martins MA, Reis AM, Carrillo MR, Guimarães $A G$, et al. Pharmaceutical care program for type 2 diabetes patients in Brazil: a randomised controlled trial. Int J Clin Pharm. 2013;35(1):79-86. http://dx.doi.org/10.1007/s11096-0129710-7. PMid:23161124.

23. Hopman WM, Harrison MB, Coo H, Friedberg $\mathrm{E}$, Buchanan $\mathrm{M}$, VanDen Kerkhof EG. Associations between chronic disease, age and physical and mental health status. Chronic Dis Can. 2009;29(3):108-16. PMid:19527569.

24. Campolina AG, Dini OS, Ciconelli RM. Impacto da doença crônica na qualidade de vida de idosos da comunidade em São Paulo (SP, Brasil). Cien Saude Colet. 2011;16(6):291925. http://dx.doi.org/10.1590/ S1413-81232011000600029. PMid:21709988. 